\title{
CONTRIBUTION CLINICO-PSYCHOLOGIQUE À L'ETUDE DE LA SCHIZOPHRENIE DES JEUNES
}

\author{
M. SCHACHTER *
}

L'affirmation faite par E. Harms (1953), prétendant que les études de M. Tramer ou de Ch. Bradley, s'efforçant de retracer les premiers symptomes de la schizophrénie infantile, à partir de "journaux" tenus soigneusement par des parents intelligents, se sont soldés par des échecs, est certainement prématurée et, en tout cas, injuste.

Il nous semble que les travaux de L. Kanner (1943-44) sur 1"“early autism", démontrent que cette voie est pleine de promesses, même si tous les cliniciens ne sont pas encore fixés sur la portée de cette entité psychiatrique. Tout récemment encore, en nous basant sur des faits personnels, nous avons, avec E. Stern (1953), souligné l'intérêt psychiatrique de l'autisme infantile, en tant que stade précurseur d'un processus dissociatif possible. Toujours en relation avec la schizophrénie infantile, nous avons relaté (1953) l'observation d'un jeune homme suivi pendant quelques années, pour des troubles caractériels et affectifs divers (énurésie, onychophagie, masturbation, tendance à tyranniser parents et animaux, vols domestiques, peurs nocturnes, manies nombreuses), dont nous n'avons pas saisi, à cette date, la signification pronostique sérieuse, avertissante, en quelque sorte. En effet, lentement, au lieu de régresser avec le temps, ces troubles ont évolué en des manifestations schizophréniques.

L'observation que nous désirons présenter plus bas est, à notre sens, un exémple frappant où l'on assiste à l'épanouissement lent, mais net, d'un comportement déjà schizophréniforme, vers un tableau clinique de schizophrénie manifeste, totale, chez un jeune homme. Evidemment, dans ce cas, un doute restera quant à la pré-existence (dans la phase pré-pubertaire, surtout) de perturbation dans le comportement ayant pu faire penser, très précocement, à une évolution possible vers une psychose.

Observons encore que ce doute quant au moment précis de l'installation de troubles pouvant être rattachés à la phénoménologie dissociative schizophrénique, largo sensu, est partagé par tous ceux qui ont l'occasion de voir ou de dépister des cas de schizophrénie des jeunes. Dans leur récent mémoire sur la schizophrénie des adolescents, Neubauer et Steinert (1952), notent combien il est, parfois, difficile de préciser si les manifestations psychonévrotiques précoces de certains enfants sont ou non des stades précoces

* Médecin-chef du Comité de l'Enfance Déficiente de Marseille (France). 
de leur schizophrénie devenue manifeste plus tard, après la puberté. On saisit aisément combien la connaissance de ces faits est importante non seulement pour le diagnostic précoce, mais aussi et surtout pour l'établissement à temps, d'un pronostic mental chez des jeunes gens consultés pour des soidisant troubles du comportement.

Observation - Jean Y., agé de 14 ans, est vu pour difficultés dans son travail scolaire, ainsi que pour quelques difficultés dans soil comportement. Dernier de 4 enfants, il est né normalement; son développement fut aussi normal; dans ses antécédents pathologiques, il faut signaler que!ques "syncopes" survenues vers 2 ou 3 ans (on aurait parlé de convulsions, mais nous n'avons pas eu des données précises); vers 10 ans, une paratyphoïlo a évolué rapidement, sans déterminations neuro-centrales. Le début de la scolarité se situe à 6 ans, sans incidents particuliers. À l'âge de 14 ans bon développement somatique; il mesure $155 \mathrm{cms}$ et pèse $44 \mathrm{~kg}$; squelette normal; rien au point de vue viscéral. Au point de vue psychologique, il s'agit d'un sujet nerveux, sensible et avec la tendance à rejeter toute autorité externe; il accepte difficilement la compagnie des autres cammarades; le sport est une chose qu'il déteste, préférant se livrer à des petites occupations avec des objets ou instruments domestiques divers. D'autre part, Jean aime lire des romans, des aventures; il fréquente aussi les films d'aventures. On le considère comme un enfant intelligent, éveillé, s'expriment de façon correcte, imagée même.

Un an plus tard, sa mère s'en plaint, en soulignant que son fils n'aime pas la société des jeunes collègues; il serait plutôt hautain, dédaigneux; manifesterait aussi une véritable haine de tout ce qui est étranger; cette xenophobie a une base objective (sa sœur ainée avait été séduite par un soldat américain qui l'a abandonnée par la suite, avec un enfant); cependant la xenophobie de Jean se généralise à tous les étrangers, sans aucune distinction. Ayant lu quelque part que les empreintes digitales servent à dépister des crimes ou autres méfaits, Jean commence par présenter un comportement assez bizarre: il se plait à saisir certains objets usuels avec un chiffon ou avec la partie latérale des doigts, afin que le dessin dactyloglyphique soit ainsi évité. C'est d'ailleurs depuis un an que Jean se masturbe en cachette; on peut se demander si ce comportement n'est en quelque sorte un moyen de se protéger contre la "découverte" de sa masturbation. Mais une autre explication doit être avancée: Jean couche très souvent dans le lit de- sa mère (cela depuis la mort de son père) invoquant des "peurs" noctırnes; peut-être des "restes" peuvent, par hasard, être découverts; aussi des "précautions" ne sont-elles pas inutiles.

À l'âge de 15 ans, son niveau au Binet-Simon est de 10 ans; cela explique probablement la puérilité du sujet. Soumis alors au test de Rorschach (Mlle. $\mathrm{S}$. Cotte), il donne 26 réponses sans refuser aucune planche. Norıs retenons dans ce test les faits essentiels que voici: $a$ ) intelligence moyenne; $b$ ) affectivité plutôt vive, mais capable de quelque auto-contrôle; c) adaptabilité pratiquement normale (rapp Vulg/Orig $=2 / 1$ ); d) existence l'une rote d'originalité un peu suspecte (réponses aux pl. 2 et 7 , où le sujet semble chercher ce qui est manifestement insolite). Mais en somme, il serait erronné de faire de ce protocole un profil manifeste de type schizophrénique. Entin, il faut noter le très fort pourcentage des réponses données en "position para-standard" (ou "rP" $\%$ ), soit $36 \%$ qui indiquait que l'on se trouve en présence d'un sujet instable, opposant, caractériel.

La scolarité de Jean n'a pas dû se développer harmonieusement car, au lycée, il a dû renoncer à l'idée de passer son baccalauréat; avart cette échéance, il quitte le lycée pour essayer d'apprendre le métier de radio-télègraphiste. Il ne peut cependant pas suivre cette instruction mi-professionnelle, mi-théorique, car cela ne l'attire pas; en fait, les maitres s'aperçoivenı que Jean n'est 
pas capable de se concentrer, ayant l'air d'être perdu durant les heures de travail. Un essai chez un relieur donne également un résultat négatif; le patron s'aperçoit assez vite que Jean n'est pas capable de se discipliner, d'exécuter soigneusement les consignes données.

Lorsque nous le voyons de nouveau, à l'âge de 18 ans 9 mois, nous avons devant nous un sujet bien bâti, presque athlétique; sa taille dépasse nettement les barêmes pour cet âge. L'examen neurologique montre des réflexes rotuliens faibles; un tremblement digital net; les épreuves cérébelleuses sont normales; la motilité et la réflectivité pupillaires sont normales. La sphère sexuelle est bien développée; la pilosité axillo-pubo-abdominale a ure disposition rigoureusement virile.

Psychiquement, l'entretien nous montre que nous avons à faire à un sujet qui est diffus, vague, fantastique; il prétend voulois approfondir la physique pour "la mettre ou l'orienter sur d'autres bases". Il prétend que ses lectures y suffisent largement; il lit des lectures consacrées à la physique nucléaire (cela dans des journaux de vulgarisation scientifique). Il s'intéresse aux voyages intersidérales, utilisant un vocabulaire où fourmillent les termes techniques qu'il ne comprend pas ou qu'il s'avère incapable de définir. Il ne sait pas au juste ce qu'il voudrait faire; prétend, de temps à autre, qu'il aimerait s'occuper de transports aériens de marchandises ce moyen étant, selon lui, le plus économique et le plus sûr, contre les voleurs. Il aimerait également devenir physicien, mais dans un sens personnel, car Jean estime que la physique officielle est trop étriquée.

Concernant le sexe opposé, Jean est formel: il déteste tout ce qui est féminin; il hait la femme, dit-il, et ajoute que pour lui il n'y a aucune exception à cette règle, même pas sa mère. Il est incapable de nous expliquer la raison ou la génèse de cette mysogynie; a nos questions pressantes, il se contente de sourire, un peu naivement. Quant à la masturbation, Jean l'avoue avec une gêne manifeste mais il atténue le fait, en voulant nous laire croire que cela lui arrive rarement. Jean nous precise qu'il n'a pas ni ne veut avoir des amis; il se plait à rester seul; d'ailleurs, il estime que les amis qu'il pourrait avoir ont des idées plates, alors que lui, il se considère comme un être évolué. Il reconnait toutefois, qu'il n'a pas encore assez de culture, mais il croit que l'auto-instruction peut y rémédier.

Nous le soumettons alors au 2e test de Rorschach. L'examen du protocole obtenu montre, de toute évidence, un changement considérable. Si l'on compare ce protocole au premier, fait à l'âge de 15 ans, les différences sautent immédiatement aux yeux: $a$ ) nombre des réponses inférieur à la normale; $b$ ) chute nette du pourcentage des morphesthésies bien viıes: soit le $\div \mathrm{F}$ tombe à $60 \%$ (antérieurement $77 \%$ ); $c$ ) la formule perceptive se rétrécit, devenant de type abstrait, le rapport G/D anormal, par rapprochement numérique des $\mathrm{G}$ aux $\mathrm{D} ; d$ ) le type de réaction subit un renversement total, passant de l'extratensivité presque égocentrique à l'introversivité pratiquement totale; c'est à dire, nous assistons à une sorte d'extinction symbolique des facteurs de la vibrance à l'unison avec l'ambiance, de la syntonie; $e$ ) le registre des contenus tout en s'étalant, voit apparaître une diminution des interprétations anthropomorphiques, comme s'il y avait un chute dans l'intérêt, ou'dans l'attention concernant l'humain; on peut même parler d'une chute, d'une sorte d'extinction, de la capacité agressive, car avec la diminution du H\% (qui passe de 24 à 10 ) il y a aussi disparition totale des réponses en position "para-standard" (réponses de type $r P$ ), symboles de cette opposition constatée dans le premier test; $f$ ) le nombre et le pourcentage des réponses originales (mal vues) augmente nettement, soulignant symboliquement le processus de désorganisation de la vie mentale; en effet, sur les 6 interprétations originales, seulement une fois, on peut parler d'une interprétation equilibrée, les autres sont assez insolites, quoique non encore franchement bizarres; $g$ ) 
l'élément puérile et immaturé est représenté, dans ce test, par lapparition à deux reprises d'interprétations de type "forme-mouvement", FK, aux planches 7 et $10 ; h$ ) il faut, enfin, mentionner à cet endroit que chez ce sujet qui se masturbe nous ne percevons qu'une seule interprétation anatomique (pl. 10) assez banale ("genre d'amygdales") à moins qu'avec les psychanalystes on admette le phénomène de déplacement "en haut", d'une préoccupation concernant un segment situé, de fait, "plus bas". Nous nous refusons cependant d'accepter cette interprétation trop facile.

Si l'on regarde globalement le protocole rorschachien obtenu, nous devons reconnaître qu'il comporte des anomalies indiscutables. Mais peut on parler d'un protocole de schizophrénie? Aurait-on posé ce diagnostic en l'absence des données cliniques ci-dessus détaillées? Il est difficile de l'affirmer. Nous laissons de côté le fait qu'aucune des réponses données par notre sujet n'a trait à des faits de séparation, de coupure, de segmentation, de "Spaltung", selon la désignation de Fr. Minkowska; bien au contraire, nous soulignons l'interprétation donnée à la pl.X (où de sujet voit "des petits singes qui s'appuient contre un objet") qui selon les vues de la même clinicienne, exprimerait la notion de "lien" (Bindung) "spécifique", soit-disant de la constitution épileptique ou épileptoide ("glyschroide" de Minkowska).

On sait que récemment èncore Piotrowski et Lewis (1950), étudiant le Rorschach dans les schizophrénies, avaient insisté sur l'apparition de quatre "signes spécifiques", à savoir: 1) des perceptions inductives, du type suivant: "cela est un œil, donc c'est une tête"; 2) des perceptions de grancleurs, du type suivant: "cela est nez long"; 3) des perceptions de fragmentation, de type: "c'est coupé en deux, etc."; 4) des jugements émotionnels de type: "cela est grotesque, compliqué, etc.". Or, dans notre $2 \mathrm{e}$ protocole, nous ne trouvons qu'une seule fois une interprétation du type inductif (à la pl. VI, notre patient dit: "c'est une peau, une descente de lit plutôt", en prenant en considération la forme et les nuances de lumière).

Pour revenir à notre discussion, nous pouvons, donc, affirmer que tout en contenant des anomalies indiscutables et des altérations nombreuses, ce 2e test ne peut pas être considéré comme typiquement schizophrénique. Or, cliniquement, nous avons vu comment est dissocié ce jeune homme, incapable depuis de très longs mois, de faire quelque chose de socialement acceptable.

Devant ces constatations, il nous a semblé plus que tentant de le soumettre au test projectif de D. J. van Lennep, connu sous la désignation de "Four picture test". Voilà, effectivement, la raison qui nous a dicté cette tentative: nous nous sommes dits que s'il est indéniable que chez notre jeune homme un processus dissociatif est en train de désorganiser sa personnalité, il doit s'avérér incapable de passer valablement ce test; que par conséquent, nous devons nous trouver dans ce cas, non pas devant une performance puérile, originale ou bizarre, mais à quelque chose de plus spécifique. Or, quelle ne fut pas notre surprise de voir se confirmer, au delà même de notre attente, notre supposition.

Lui ayant présenté les 4 images du test de van Lennep, après les explications nécéssaires, Jean regarde attentivement et nous dit qu'il saisit fort bien ce que nous demandons, qu'il saisit également le sens de chacune des images, mais refuse de voir la moindre possibilité de les réunir pour en faire une seule histoire; il est catégorique lorsqu'il dit "c'est absurde vouloir faire ce que vous me demandez; cela n'est pas possible"; il rest sur ces positions, malgré nos suggestions et encouragements; la chose lui apparaît donc jugée ainsi qu'il le décide. La seule concession qu'il nous fait c'est de nous décrire séparément les 4 images; la 1ère, une scène de dispute entre patron et employé; la 2e, une chambre sale avec un lit infecte; la $3 e$, une prostituée, "mais quelle idée, faire le trottoir sous la pluie"; la 4e, une partie de tennis. 
En somme, dans le test de van Lennep, nous avons, de façon indiscutable, a la lumière des procédés psychologiques projectifs, un fait plaidant pour la nature schizophrénique de la perturbation de base dont est atteint notre patient.

Nous ne voulons pas discuter ici la validité du test de Rorschach ou de van Lennep, dans le diagnostic des syndromes schizophréniques en général. Nous pensons le faire une autre fois, en apportant l'ensemble de notre matériel. Nous nous contentons, par conséquent, à l'exposé de notre cas, sans aucune autre discussion supplémentaire.

Nous devons ajouter encore un court commentaire concernant la conduite thérapeutique. On nous avait demandé, en nous adressant, dernièrement, notre jeune patient, s'il y avait lieu de suivre le conseil donné par un jeune psychiytre, et d'envisager une cure de Sackel. Notre réponse a été de nous opposer formellement à cette thérapeutique. Nous n'avons, effectivement, aucune raison de croire que ce traitement insulinique (ou tout autre choc biologique) donne le succès désiré. Nous sommes, c'est l'évidence même, devant un cas léger, mais invétéré, et l'expérience est assez riche, qui nous montre le peu de résultats solides que ces cures permettent de réaliser. Par contre, nous nous sommes prononcés en faveur d'une cure psychothérapique, combinée avec une instruction professionnelle pratique, en donnant la préférence à un métier en rapport avec les capacités intellectuelles, moyennes, de notre patient. Nous ne nous cachons pas du tout l'énorme difficulté qui consiste à trouver un artisan qui soit en même temps le maître et le soutien affectueux de notre jeune patient. De plus, nous ne nous cachons, pas, non plus, le fait que ce genre de travail combiné avec la supervision psychothérapique, demande beaucoup de temps et beaucoup de patience. Mais, ne s'agit-il pas, dans des cas comme le notre, de tout mettre en œuvre pour obtenir, au prix de toutes ces difficultés, une réintégration, même partielle.

\section{RẼSUME}

Nous présentons l'observation clinique et psychologique d'un jeune homme atteint d'un syndrome schizophrénique; l'étude montre qu'à la puberté il présentait déjà une série de manifestations rentrant dans la sphère du comportement schizophréniforme. Alors, l'examen clinique laissait la porte ouverte à des interprétations libérales, quant à la validité du diagnostic; on aurait pu considérér alors le jeune pubertaire comme un caractériel ou comme un sujet plus ou moins original. Au test de Rorschach pratiqué à ce moment, l'ensemble des constatations faites démontrait l'existence indiscutable d'un attachement net avec l'ambiance. Par contre, à l'âge de 18 ans 9 mois, le tableau schizophrénique est cliniquement indiscutable. Au Rorschach on note des modifications très nombreuses, très significatives (surtout en ce qui concerne la dissolution et la disparition des facteurs de la syntonie). Cependant, le test ne présente pas les caractères considérés comme "spécifiques" de la schizophrénie. C'est, par contre, dans le test projectif de van Lennep que la nature dissociative du tableaux clinique fut démontrée de façon claire et nette. 


\section{SUMMARY}

Clinico-physiological report of the case of a young man with schizophrenic symptoms; the study showed that this patient exhibited some schizophreniform manifestations at the puberty but at this moment the diagnosis was not ascertained, being considered as of neurotic origin. The Rorschachtest then performed was normal and exhibited syntonic patterns. Conversely, at 18 years 9 months, the clinical picture is of schizophrenic frame; the Rorschach-test shows many abnormalities with the fading of syntonic symptoms; however, the pattern was not "specifically" schizophrenic. At the van Lennep "four picture test" there was a picture of a dissociative disturbance in the sense of schizophrenia.

\section{BIBLIOGRAPHIE}

HARMS, E. - Essentials of Abnormal Child Psychology. The Julian Press, Publishers, New York, 1953. KORNFELD, M. - The development of schizophrenic symptoms in young children. The Nervous Child., 10:112, 1952. MARGULIES-MEHR, H. - The application of psychological tests and methods to schizophrenia in children. The Nervous Child., 10:63, 1952. MULLER-HEGEMANN, D. - Die Psychoterapie bei schizophrenen Prozessen. Edit. S. Hirzel, Leipzig, 1952. NEUBAUER, P. B. - Schizophrenia in adolescence. The Nervous Child., 10:129, 1952. PIOTROWSKI, Z. A. et LEWIS, N. D. C. - An experimental Rorschach diagnostic aid for some forms of schizophrenia. Am. J. Psychiat., 107:360, 1950. SCHULTZ-HENCKE, H. — Das Problem des Schizophrenie. Georg Thieme, Stuttgart, 1952. TRAMER, M. - Lehrbuch der allgemeinen Kinderpsychiatrie, ed. 3. Edit. B. Schwabe \& Co., Basel, 1949.

40A, Bd. Voltaire - Marseille, France. 